\title{
Response to the letter to the editor by Shek et al. on Vergeldt et al: Risk factors for pelvic organ prolapse and its recurrence: a systematic review
}

\author{
Tineke F. M. Vergeldt ${ }^{1} \cdot$ Mirjam Weemhoff $^{2} \cdot$ Joanna IntHout $^{3} \cdot$ Kirsten B. Kluivers $^{1}$
}

Received: 8 January 2016 / Accepted: 8 January 2016 / Published online: 6 February 2016

(C) The International Urogynecological Association 2016

\section{Dear Editor,}

We thank Ka Lai Shek and Hans Peter Dietz for their interest in our systematic review [1]. They state in their comment that we have come to an erroneous conclusion by setting arbitrary inclusion criteria and by ignoring some studies all together. We disagree with this statement and would like to explain this further.

Because of the enormous amount of articles published on risk factors for pelvic organ prolapse (POP) and POP recurrence, we were able to apply strict inclusion criteria in our systematic review, to select only the strongest available evidence. Because of this strategy, risk factors with a low prevalence that can only be examined in case-control studies, such as collagen and matrix metalloproteinase polymorphisms, have been missed in our systematic review. In our discussion we have described this as a limitation of our study.

Nonetheless, pelvic floor factors such as levator ani muscle defects and the size of the levator ani hiatus, are not rare $[2,3]$. Thus, they can easily be investigated as risk factors for POP or POP recurrence in cohort studies or cross-sectional studies. To prevent bias in these types of studies, it is important to control for potential confounders, for example, by stratification or multivariate analysis. To select only strong evidence, we

This reply refers to the comment available at doi:10.1007/s00192-016-2953-4.

Tineke F. M. Vergeldt

tinekevergeldt@gmail.com

1 Department of Obstetrics and Gynecology, Radboud University Medical Center, P.O. Box 9101, 6500HB Nijmegen, The Netherlands

2 Department of Obstetrics and Gynecology, Atrium Medical Center Parkstad, Heerlen, The Netherlands

3 Department for Health Evidence, Radboud University Medical Center, Nijmegen, The Netherlands included only articles in which confounders were taken into account, i.e., in which a multivariate analysis was performed. In addition, we have only defined a variable as a confirmed risk factor in case it was significantly associated with POP or POP recurrence in at least two studies.

Shek et al. state that there is "quite substantial evidence" that levator defects and the levator hiatal area are risk factors for POP and POP recurrence. Nonetheless, among the articles with the strongest available evidence selected for our systematic review only one study showed a significant association between levator defects and POP and the levator hiatal area and POP, and only one study showed a significant association between levator defects and POP recurrence. There were no other studies that met the inclusion criteria to confirm these results. In addition, Shek et al. state that we "ignored some studies all together". As they showed in the table they have provided, none of these articles met our inclusion criteria. This supports our claim that our search was thorough and complete.

Finally, the statement that our conclusion concerning pelvic floor factors is erroneous is not supported by the articles with the strongest available evidence on this subject. We have provided the highest level of evidence possible, to prevent bias and to provide undisputable evidence. More high level evidence that would expand the results of our systematic review would be appreciated.

\section{References}

1. Vergeldt TFM, Weemhoff M, IntHout J, Kluivers K (2015) Risk factors for pelvic organ prolapse and its recurrence: a systematic review. Int Urogynecol J 26:1559-1573

2. Dietz HP, Steensma AB (2006) The prevalence of major abnormalities of the levator ani in urogynaecological patients. BJOG 113:225-230

3. Dietz HP, Simpson J (2008) Levator trauma is associated with pelvic organ prolapse. BJOG 115:979-984 